\title{
Towards understanding of glycaemic index and glycaemic load in habitual diet: associations with measures of glycaemia in the Insulin Resistance Atherosclerosis Study
}

\author{
Elizabeth J. Mayer-Davis ${ }^{1}$, Ashish Dhawan ${ }^{1}$, Angela D. Liese ${ }^{1}$, Karen Teff $^{2}$ and Mandy Schulz ${ }^{3}$ \\ ${ }^{1}$ Center for Research in Nutrition and Health Disparities and Department of Epidemiology and Biostatistics, University of South \\ Carolina Arnold School of Public Health, 2718 Middleburg Drive, Columbia, SC 29208, USA \\ ${ }^{2}$ National Institutes of Health, National Institute of Diabetes, Digestive, and Kidney Diseases, Bethesda, MD 20817, USA \\ ${ }^{3}$ German Institute of Human Nutrition, Department of Epidemiology, Potsdam-Rehbruecke, Germany
}

(Received 8 June 2005 - Revised 19 September 2005 - Accepted 28 September 2005)

\begin{abstract}
Epidemiologic studies have applied the glycaemic index (GI) and glycaemic load (GL) to assessments of usual dietary intake. Results have been inconsistent particularly for the association of GI or GL with diabetes incidence. We aimed to advance understanding of the GI and GL as applied to food frequency questionnaires (FFQ) by evaluating GI and GL in relation to plasma measures of glycaemia. Included were 1255 adults at a baseline examination (1994-6) and 813 who returned for the 5-year follow-up examination. Usual diet, at both examinations, was assessed by a validated FFQ. GI and GL were evaluated in relation to average fasting glucose (two measures at each examination) and $2 \mathrm{~h}$ post- $75 \mathrm{~g}$ glucose load plasma glucose (baseline and follow-up), and glycated haemoglobin (A1c; follow-up only); using generalized linear models. Correlation coefficients $(r)$ for GI and GL related to measures of glycaemia, adjusted for total energy intake, ranged from - 0.004 to 0.04 (all NS) for both examinations. Adjustment for potential confounders, for fasting glucose in models for $2 \mathrm{~h}$ glucose (to model incremental glucose) and for average fasting glucose in models for A1c (to account, in part, for overnight endogenous glucose production) also did not materially alter findings, nor did inclusion of data from both examinations together in linear mixed models. The present results call into question the utility of GI and GL to reflect glycaemic response to food adequately, when used in the context of usual diet. Further work is needed to quantify usual dietary exposures relative to glucose excursion and associated chronic glycaemia and other metabolic parameters.
\end{abstract}

Dietary assessment: Habitual diet: Epidemiologic studies

Glycaemic index (GI) and glycaemic load (GL) have been incorporated into food frequency questionnaire (FFQ)-based assessments of usual diet in large epidemiological studies; however, associations of GI and GL with diabetes incidence have been reported inconsistently. Two large prospective studies were negative for GI and GL (Meyer et al. 2000; Stevens et al. 2002), one was positive for both GI and GL (Salmeron et al. 1997a), two were positive for GI but negative for GL (Salmeron et al. 1997b; Schulze et al. 2004), and one was positive for GI (Hodge et al. 2004).

Reasons for inconsistent findings are not well understood and may relate to the underlying concept of GI and GL when used in the context of usual dietary intake rather than single food or single meal effects. The premise of GI is that glucose excursion due to ingestion of a carbohydrate-containing food, relative to that of a standard food, is fairly constant across individuals and thus can be quantified as GI for that food (Jenkins et al. 1981; Wolever et al. 1991). GI values currently utilized have been determined based on glucose excursion $2 \mathrm{~h}$ after ingestion of test foods or meals administered in the fasting state (Foster-Powell et al. 2002) to non-diabetic individuals. In contrast, usual dietary intake involves the cumulative exposure to food consumed throughout the day, with some meals or individual foods consumed prior to glucose returning to the basal state. And, usual diet summarizes intake over a period of several months or (commonly) 1 year. These distinctions may be highly relevant to understanding GI as applied to usual diet for three reasons. First, macronutrients in mixed meals often elicit glucose and insulin excursions well beyond the $2 \mathrm{~h}$ used to establish the GI (Gannon \& Nuttall, 1987; Nuttall \& Gannon, 1991), thus chronic physiological effects of mixed meals may not be captured adequately by the GI when applied to usual diet. Second, it has been shown that glycaemic response to the same meal consumed serially through the day (4h apart) can vary considerably (Nuttall et al. 1985; Gannon \& Nuttall, 1987; Ercan et al. 1994); this is not considered in the application of GI to assessment of usual diet by FFQ in epidemiological studies. Third, debate continues regarding the impact of dietary protein and fat in the overall diet on glycaemia

Abbreviations: A1c, glycated haemoglobin; FFQ, food frequency questionnaire; GI, glycaemic index; GL, glycaemic load; IRAS, Insulin Resistance Atherosclerosis Study; OGTT, oral glucose tolerance test.

* Corresponding author: Dr Elizabeth J. Mayer-Davis, fax +1 803251 7873, email EJMayer@gwm.sc.edu 
(Nuttall \& Gannon, 1991; Eckel, 2003; Gannon et al. 2003; Gannon \& Nuttall, 2004), and several methodological limitations of relevant work to date have been noted (Eckel, 2003).

The goal of the present analysis was to elucidate the meaning of GI in the context of an assessment of usual dietary intake, and thereby to shed light on the inconsistent findings of GI and GL in relation to diabetes incidence. Because GI is based on glucose excursion resulting from ingestion of food, we considered that GI of a usual diet measured in a large cohort of adults should be associated with inter-individual variation in various measures of glycaemia. In particular, we hypothesized that GI and GL of the usual diet would be positively associated with glycated haemoglobin (A1c), which provides a weighted average of glycaemia over approximately the preceding 3 months (Tahara \& Shima, 1995). We based this hypothesis on the finding that acarbose, which pharmacologically slows carbohydrate digestion, is effective in lowering A1c in a dose-dependent fashion (Coniff et al. 1994; Bischoff, 1995). Similarly, usual diets that are lower in GI would be expected to be more slowly digested and absorbed (Jenkins et al. 1981; Wolever et al. 1991) and would thus be associated with lower A1c. Analyses were conducted in the multi-ethnic, adult participants in the Insulin Resistance Atherosclerosis Study (IRAS), for whom usual diet was assessed by a FFQ validated within the study population (Mayer-Davis et al. 1999b).

\section{Research design and methods}

\section{Subject selection}

The design of IRAS has been described in detail elsewhere (Wagenknecht et al. 1995). Participants were recruited at four clinical centres between 1992 and 1994 for the IRAS baseline examination. The goal was to obtain nearly equal representation of participants across glucose tolerance status (normal glucose tolerance; impaired glucose tolerance; noninsulin-taking type 2 diabetes mellitus); race/ethnicity (African American, Hispanic and non-Hispanic white); sex; and age (40-49, 50-59, 60-69 years). Race/ethnicity was determined by self-report using 1990 US census questions. The final sample comprised 1625 people, of whom $38 \%$ were non-Hispanic white, $34 \%$ Hispanic and $29 \%$ African American. Normal glucose tolerance was present in $44.4 \%, 22.7 \%$ had impaired glucose tolerance and $33.1 \%$ had type 2 diabetes. All participants provided written informed consent as approved by their respective field centre's institutional review board.

A follow-up visit occurred during 1997-9 (average followup was 5.2 years, range $4.5-6.6$ years), and 1313 participants $(81 \%)$ returned. Those who attended the follow-up examination were similar to those who did not attend in terms of ethnicity, sex, baseline glucose tolerance status (normal glucose tolerance $v$. impaired glucose tolerance), and BMI (all comparisons, $P>0 \cdot 3$ ) (Hanley et al. 2002).

\section{Data collection}

For both the baseline and the 5-year follow-up examinations, IRAS required a two-visit protocol, the first to determine glucose tolerance status and the second to measure insulin sensitivity. Participants were asked to fast for $12 \mathrm{~h}$ prior to each of the two visits, abstain from heavy exercise and alcohol for $24 \mathrm{~h}$, and refrain from smoking on the morning of the visit. From visit 1, a $2 \mathrm{~h}, 75 \mathrm{~g}$ oral glucose tolerance test (OGTT; Orange-dex, Custom Laboratories, Baltimore, MD, USA) was performed during the first visit, and WHO criteria (World Health Organization, 1985) were used to assign glucose tolerance status. Individuals currently taking oral hypoglycaemic medications were classified as having type 2 diabetes regardless of the results of the OGTT. Average fasting glucose (from the two visits), $2 \mathrm{~h}$ postprandial glucose from the OGTT ( $2 \mathrm{~h}$ glucose) and A1c were the outcome variables of interest for the present report. Average fasting glucose and $2 \mathrm{~h}$ glucose were obtained at both the baseline and the 5year follow examinations. Plasma glucose concentration was measured in duplicate using the glucose oxidase technique on an autoanalyser (Yellow Springs Equipment Co., Yellow Springs, OH, USA). A1c, obtained only at the 5-year follow-up examination, was measured by automated microparticle immunoassay using whole blood (Penn Medical Laboratory, Medlantic Research Institute, Washington, DC, USA). Anthropometric measures were taken with the participant in lightweight clothing with shoes removed. Height and weight were measured in duplicate and recorded to the nearest $0.5 \mathrm{~cm}$ and $0.1 \mathrm{~kg}$, respectively. BMI was calculated as weight $/$ height $^{2}\left(\mathrm{~kg} / \mathrm{m}^{2}\right)$. Usual frequency of vigorous physical activity was ascertained in an interviewer-administered, 1-year activity recall that incorporated activities current among IRAS participants (Mayer-Davis et al. 1998).

Usual intake of diet was assessed by interview using a 1year, semi-quantitative 114-item food frequency interview, modified from the National Cancer Institute Health History and Habits Questionnaire, to include regional and ethnic food choices across the four clinical centres (Mayer-Davis et al. 1999b). Participants were asked to recall intake of foods and beverages over the past year. Interviewers were centrally trained and certified, and audiotapes of interviews were reviewed quarterly. The validity and reproducibility of the IRAS FFQ to measure nutrient intake has been demonstrated in a subset of the IRAS population (Mayer-Davis et al. $1999 b$ ). Daily nutrient intake was estimated from the FFQ using an expanded nutrient database (HHHQ-DIETSYS analysis software, version 3.0; National Cancer Institute, Bethesda, MD, 1993; supplemented with information from the Minnesota Nutrition Data System PC System, Program 2.3; Nutrition Coordinating Center, 1990). Intake of alcoholic beverages was evaluated separately using a frequency approach with additional questions about recent use and average lifetime use.

\section{Estimation of dietary glycaemic index and glycaemic load}

We assigned mean GI values based on the white bread standard from published data (Foster-Powell et al. 2002) and other available resources (T. M. S. Wolever, personal communication) to all 114 FFQ line items plus three items assessed in the examination 1 interview on alcohol consumption (beer, wine, liquors) plus several additional foods that were identified in open-ended questions as being consumed more than once per week. Where multiple foods were assessed in a single FFQ line, we assigned a GI value that was the weighted average of the food-specific GI values. 
The weighting factors were determined based on the prevalence of estimated population consumption of those items (Block et al. 1986; Mayer-Davis et al. 1999b). Food items containing minute amounts of carbohydrates (e.g. meat, fish and some vegetables) were assigned a GI value of 100 (the GI of white bread, based on the white bread standard), which is assumed to not affect the estimated daily GI due to small amounts of carbohydrates consumed with these foods (Jenkins et al. 1994). Cheese was assigned the GI of milk. All GI values presented here are based on the white bread standard because a majority of papers from the epidemiological literature to date have used the white bread standard (rather than the glucose standard). Average dietary GI and GL were computed as follows. Note that computation included digestible carbohydrate only (calculated as total carbohydrate minus fibre) because only digestible carbohydrates are considered in establishing GI values.

$$
\mathrm{GI}=\sum_{\text {items }}[(\text { Digestible carbohydrate/Serving of item }
$$

$$
\times \text { Average number of servings } / \mathrm{d} \text { ) }
$$

$\times$ Item GI]/Total digestible carbohydrate intake

$$
\mathrm{GL}=\sum_{\text {items }}[(\text { Digestible carbohydrate/Serving of item }
$$

$$
\times \text { Average number of servings } / \mathrm{d}) \times \text { Item GI] } / 100
$$

\section{Statistical analysis}

Of the 1625 individuals included in the final IRAS cohort at baseline and 1313 who returned for the follow-up examination, these analyses were limited to 1295 individuals at visit 1 and 862 at visit 2 who had either normal or impaired glucose tolerance, or who had previously undiagnosed diabetes. This permitted a reasonably wide range of glycaemia, while avoiding individuals being treated for diabetes or those with increased glycaemia due to the marked increase in glucose production by the liver known to occur in longstanding diabetes. Individuals with a known diagnosis of diabetes were excluded on the premise that knowledge of the condition might have altered their dietary behaviour, potentially biasing study findings. Also excluded were participants with missing data on the outcome variables, dietary variables and covariates, resulting in a final sample size of 1255 for the baseline examination analyses, and 813 for the follow-up examination analyses. Further detail regarding minor sample size reductions due to occasional missing data for various models is provided in the tables.

Dietary variables considered as main exposures included GI and GL. Dietary carbohydrate, fibre, starch, fructose and glucose were also evaluated as a secondary analysis. Unadjusted association between GI or GL and measures of glycaemia were assessed by a one-way ANOVA, where differences between the means of the outcomes by GI or GL quintiles were evaluated. The association was further explored by correlations between the dietary variables and outcomes adjusted for total energy intake. Subsequent analysis used generalized linear models for analysis. Covariates for this set of analyses were total energy intake, demographic variables (age, sex and ethnicity/clinic), lifestyle factors (alcohol intake, current smoking and vigorous activity), BMI and family history of diabetes. Because family history did not contribute appreciably to the models (after adjustment for all other covariates), models presented herein were not adjusted for this variable. Finally, other macronutrients were added to the models (dietary fat and protein) to adjust for potential confounding by these variables.

For the outcomes $2 \mathrm{~h}$ glucose and A1c, we also adjusted for fasting glucose as follows. For $2 \mathrm{~h}$ glucose, we wished to evaluate the potential contribution of GI or GL of a usual diet to inter-individual differences in incremental glucose excursion after a standard load, therefore we adjusted for fasting glucose from the OGTT and refer to this result in terms of $2 \mathrm{~h}$ incremental glucose. For the outcome A1c, it was recognized that A1c reflects chronic glycaemia over time, incorporating both endogeneous glucose production and postprandial response. To account, at least in part, for the contribution of overnight glucose production to chronic glycaemia captured in A1c, the average fasting glucose (from the two visits at the follow-up examination) was entered into the final model.

Finally, data from both examinations were pooled in linear mixed models (Littell et al. 2000) in order to maximize statistical precision for associations of GI and GL with measures of glycaemia (other than for A1c, which was available only at the second examination). This approach incorporated a total of 2071 observations. Regarding criteria for statistical significance, adjustments to statistical testing to account for multiple comparisons generally assume that the tests are independent of each other (Perneger, 1998). In this paper, by design, we employed several measures of glycaemia as outcome measures; thus inherently, the tests performed were not independent of each other. Therefore, adjustments for multiple comparisons were not made and the traditional criteria for statistical significance $(P<0.05)$ was used. All analyses were performed using SAS version 8.2 (SAS Institute Inc., Cary, NC, USA).

\section{Results}

Descriptive characteristics of the study participants at the baseline and follow-up examinations are shown in Table 1. Mean GI at baseline and follow-up was 82.66 (SD 5.59) and 82.48 (SD 5.31), respectively. GL was 181.54 (SD 80.81) at baseline and was 171.36 (SD 80.92) at follow-up. At baseline, percentage energy from carbohydrate and fat were 49.1 (SD 8.5 ) and 33.5 (SD 7.2), respectively. Table 2 shows measures of glycaemia (unadjusted) according to quintile of GI or GL, for each examination. No statistically significant associations were observed.

Correlation coefficients for associations of dietary variables, adjusted only for total energy intake, with measures of glycaemia are presented in Table 3. No statistically significant associations were observed for any measure of glycaemia in relation to either GI or GL, at either examination. Several statistically significant associations were seen for carbohydraterelated nutrients in relation to measures of glycaemia. Lower intake of starch and fibre was associated with higher average fasting glucose at both examinations; however, statistical significance $(P<0.05)$ was reached only at baseline for starch, 
Table 1. Descriptive characteristics of participants in the Insulin Resistance and Atherosclerosis Study at baseline $(n$ 1255) and follow-up ( $n$ 813) examinations*

\begin{tabular}{|c|c|c|c|c|}
\hline \multirow[b]{2}{*}{ Characteristics } & \multicolumn{2}{|c|}{ Baseline (1994-6) } & \multicolumn{2}{|c|}{ Follow-up (1998-9) } \\
\hline & Mean or \% & SD & Mean or $\%$ & SD \\
\hline \multicolumn{5}{|l|}{ Dependent variables } \\
\hline Average fasting glucose $(\mathrm{mg} / \mathrm{dl}) \dagger$ & $104 \cdot 03$ & $26 \cdot 71$ & $101 \cdot 16$ & $18 \cdot 19$ \\
\hline Two-hour postprandial glucose (mg/dl) & $147 \cdot 88$ & $65 \cdot 85$ & 140.51 & $54 \cdot 20$ \\
\hline Glycated haemoglobin (\%)‡ & NA & & $5 \cdot 39$ & 0.69 \\
\hline \multicolumn{5}{|l|}{ Independent variables } \\
\hline Glycaemic index & $82 \cdot 66$ & 5.59 & $82 \cdot 48$ & $5 \cdot 31$ \\
\hline Glycaemic load & 181.54 & $80 \cdot 81$ & $171 \cdot 36$ & $80 \cdot 92$ \\
\hline Dietary total energy (kJ) & $8163 \cdot 90$ & $3530 \cdot 54$ & $7897 \cdot 30$ & $3642 \cdot 17$ \\
\hline Dietary carbohydrate (g) & $235 \cdot 77$ & $100 \cdot 50$ & 222.92 & $102 \cdot 77$ \\
\hline Dietary fibre $(\mathrm{g})$ & $16 \cdot 83$ & $7 \cdot 96$ & $15 \cdot 64$ & $8 \cdot 21$ \\
\hline Dietary starch (g) & $98 \cdot 73$ & $52 \cdot 01$ & $92 \cdot 62$ & $52 \cdot 11$ \\
\hline Dietary fructose (g) & $27 \cdot 44$ & $17 \cdot 69$ & $26 \cdot 15$ & $16 \cdot 67$ \\
\hline Dietary glucose (g) & $24 \cdot 86$ & $16 \cdot 38$ & 24.00 & $15 \cdot 36$ \\
\hline Dietary fat $(\mathrm{g})$ & 74.43 & 39.63 & $75 \cdot 20$ & $42 \cdot 39$ \\
\hline Dietary protein (g) & $79 \cdot 46$ & $35 \cdot 60$ & $77 \cdot 19$ & 37.86 \\
\hline BMI $\left(\mathrm{kg} / \mathrm{m}^{2}\right)$ & 29.05 & 5.91 & 28.89 & $5 \cdot 76$ \\
\hline Age (years) & $55 \cdot 32$ & $8 \cdot 50$ & $59 \cdot 76$ & 8.45 \\
\hline Gender (male) & $43.51 \%$ & & $42.68 \%$ & \\
\hline \multicolumn{5}{|l|}{ Ethnicity } \\
\hline Non-Hispanic white & $39.28 \%$ & & $40.84 \%$ & \\
\hline African American & $28.21 \%$ & & $25.95 \%$ & \\
\hline Hispanic & $32.51 \%$ & & $33.21 \%$ & \\
\hline \multicolumn{5}{|l|}{ Alcohol intake } \\
\hline Never drank & $11.87 \%$ & & $10.09 \%$ & \\
\hline Ex-drinker & $14 \cdot 10 \%$ & & $16 \cdot 36 \%$ & \\
\hline Occasional & $17 \cdot 77 \%$ & & $18.70 \%$ & \\
\hline$\leq \frac{1}{2} \mathrm{drink} / \mathrm{d}$ & $29.16 \%$ & & $29.77 \%$ & \\
\hline$\frac{1}{2}-<1$ drink/d & $9 \cdot 32 \%$ & & $8.49 \%$ & \\
\hline $1-<3$ drinks/d & $13 \cdot 31 \%$ & & $13.53 \%$ & \\
\hline$\geqq 3$ drinks/d & $4.46 \%$ & & $3.08 \%$ & \\
\hline Positive family history of diabetes & $42 \cdot 23 \%$ & & $43.42 \%$ & \\
\hline \multicolumn{5}{|l|}{ Diabetic status } \\
\hline Normal & $56 \cdot 25 \%$ & & $58.67 \%$ & \\
\hline Impaired glucose tolerance & $27.89 \%$ & & $28.66 \%$ & \\
\hline Diabetic (previously undiagnosed) & $15.86 \%$ & & $12 \cdot 67 \%$ & \\
\hline \multicolumn{5}{|l|}{ Smoking status } \\
\hline Never & $45.26 \%$ & & $48.09 \%$ & \\
\hline Past & $38.01 \%$ & & $42 \cdot 80 \%$ & \\
\hline Current & $16 \cdot 73 \%$ & & $9.10 \%$ & \\
\hline \multicolumn{5}{|l|}{ Frequency of vigorous activity } \\
\hline Rarely or never & $29.80 \%$ & & $31.00 \%$ & \\
\hline $1-3$ times/month & $19 \cdot 84 \%$ & & $19.93 \%$ & \\
\hline 1 time/week & $12.99 \%$ & & $14.76 \%$ & \\
\hline 2-4 times/week & $27.57 \%$ & & $23.62 \%$ & \\
\hline$\geqq 5$ times/week & $9.80 \%$ & & $10 \cdot 70 \%$ & \\
\hline
\end{tabular}

NA, not applicable.

${ }^{*}$ For details of procedures, see p. 398.

† Average of two values of fasting plasma glucose measured 1 week to 1 month apart. Due to missing values, sample size for baseline examination was 1218 and 775 for follow-up examination.

‡Due to missing values, sample size for analysis with glycated haemoglobin as outcome variable was 775 .

and only at follow-up for fibre. Higher starch intake was associated with greater incremental $2 \mathrm{~h}$ glucose $(2 \mathrm{~h}$ postprandial glucose adjusted for fasting glucose, $P<0.05$ only at follow-up), and higher intake of fructose and glucose was associated with lower incremental $2 \mathrm{~h}$ glucose at follow-up $(P<0.05)$. Dietary fibre intake was inversely associated with A1c $(r-0.08, P<0.05)$.

Table 4 shows results of regression modelling for each measure of glycaemia, sequentially adjusted for covariates. GI was not associated with any measure of glycaemia at either examination. For average fasting glucose, inverse associations comparable in magnitude were observed for GL and total carbo- hydrate, although associations were attenuated and were no longer statistically significant after adjustment for lifestyle factors (at follow-up, Table 4, model 2) or for BMI (at baseline, Table 4, model 3). After adjustment for demographic variables, lifestyle factors and BMI, dietary starch tended to be inversely associated with average fasting glucose $(0.05 \leq P<0.10$ for both examinations, Table 4, model 3) as was dietary fibre $(0.05 \leq P<0.10$ for follow-up, Table 4 , model 3$)$. Dietary glucose tended to be inversely associated with $2 \mathrm{~h}$ postprandial glucose, after adjustment for covariates $(0.05 \leq P<0 \cdot 10$; Table 4 , model 3). For $2 \mathrm{~h}$ incremental glucose (Table 4 , model 4 for $2 \mathrm{~h}$ postprandial glucose adjusted for fasting glucose), dietary 
Table 2. Mean values for various measures of glycaemia by glycaemic index and glycaemic load quintiles (I-V) of participants in the Insulin Resistance and Atherosclerosis Study at baseline $\left(n\right.$ 1255) and follow-up $(n 813)^{*}$

\begin{tabular}{|c|c|c|c|c|c|c|c|c|c|c|c|}
\hline & \multicolumn{2}{|c|}{ I } & \multicolumn{2}{|c|}{ ॥ } & \multicolumn{2}{|c|}{ III } & \multicolumn{2}{|c|}{ IV } & \multicolumn{2}{|c|}{$\mathrm{v}$} & \multirow[b]{2}{*}{$P$ valuet } \\
\hline & Mean & SE & Mean & SE & Mean & SE & Mean & SE & Mean & SE & \\
\hline \multicolumn{12}{|l|}{ Glycaemic index } \\
\hline Baselineł & 74.92 & $2 \cdot 78$ & 79.83 & 0.94 & 82.69 & 0.75 & 85.45 & 0.87 & 90.39 & $3 \cdot 28$ & \\
\hline Follow-up & 75.08 & 3.04 & 79.91 & 0.80 & 82.52 & 0.74 & 85.19 & 0.77 & 89.72 & 2.93 & \\
\hline \multicolumn{12}{|c|}{ Average fasting glucose $(\mathrm{mg} / \mathrm{dll}) \S$} \\
\hline Baseline ( $n$ 1218) & $106 \cdot 12$ & 1.71 & 103.58 & 1.71 & $104 \cdot 71$ & 1.71 & $102 \cdot 83$ & 1.71 & 102.89 & $1 \cdot 71$ & NS \\
\hline Follow-up ( $n 775$ ) & $102 \cdot 11$ & 1.46 & 99.86 & 1.46 & 101.91 & 1.46 & 101.69 & 1.46 & $100 \cdot 20$ & 1.46 & NS \\
\hline \multicolumn{12}{|c|}{ 2-h postprandial glucose $(\mathrm{mg} / \mathrm{dl})$} \\
\hline Baseline ( $n$ 1255) & $153 \cdot 24$ & 4.15 & 142.94 & 4.15 & 156.06 & $4 \cdot 15$ & 141.98 & $4 \cdot 15$ & $145 \cdot 19$ & $4 \cdot 15$ & 0.0518 \\
\hline Follow-up ( $n 813)$ & 146.09 & 4.25 & $135 \cdot 35$ & 4.25 & $141 \cdot 25$ & 4.26 & 139.73 & 4.25 & $140 \cdot 13$ & 4.26 & NS \\
\hline \multicolumn{12}{|c|}{ Glycated haemoglobin (\%)\| } \\
\hline Follow-up ( $n 775)$ & 5.40 & 0.06 & $5 \cdot 38$ & 0.06 & $5 \cdot 38$ & 0.06 & $5 \cdot 38$ & 0.06 & 5.41 & 0.06 & NS \\
\hline \multicolumn{12}{|l|}{ Glycaemic load } \\
\hline Baseline & $86 \cdot 72$ & 19.67 & 133.03 & $10 \cdot 13$ & $170 \cdot 85$ & $12 \cdot 19$ & 212.06 & 12.75 & 305.02 & 64.83 & \\
\hline Follow-upł & $81 \cdot 11$ & 18.59 & 123.24 & $9 \cdot 3$ & 158.51 & 10.55 & 198.67 & 13.25 & 295.96 & 71.90 & \\
\hline \multicolumn{12}{|c|}{ Average fasting glucose $(\mathrm{mg} / \mathrm{dll}) \S$} \\
\hline Baseline ( $n$ 1218) & 103.56 & 1.71 & 103.01 & 1.71 & $106 \cdot 40$ & 1.71 & 103.75 & 1.71 & 103.41 & 1.71 & NS \\
\hline Follow-up ( $n 775)$ & 101.35 & 1.46 & $101 \cdot 16$ & 1.46 & 102.05 & 1.46 & $100 \cdot 82$ & 1.46 & $100 \cdot 40$ & 1.46 & NS \\
\hline \multicolumn{12}{|c|}{ 2-h postprandial glucose $(\mathrm{mg} / \mathrm{dl})$} \\
\hline Baseline ( $n$ 1255) & 149.47 & $4 \cdot 16$ & $148 \cdot 80$ & 4.16 & 152.43 & $4 \cdot 16$ & $144 \cdot 88$ & $4 \cdot 16$ & 143.82 & $4 \cdot 16$ & NS \\
\hline Follow-up ( $n 813)$ & $143 \cdot 26$ & 4.25 & $138 \cdot 26$ & 4.25 & 142.64 & $4 \cdot 26$ & $135 \cdot 82$ & 4.25 & 142.59 & $4 \cdot 26$ & NS \\
\hline \multicolumn{12}{|c|}{ Glycated haemoglobin $(\%) \|$} \\
\hline Follow-up $(n 775)$ & 5.43 & 0.06 & $5 \cdot 38$ & 0.06 & 5.37 & 0.06 & $5 \cdot 38$ & 0.06 & $5 \cdot 38$ & 0.06 & NS \\
\hline
\end{tabular}

${ }^{\star}$ For details of procedures, see p. 398

† $P$ value is of the $F$-test for difference between means from the one-way ANOVA procedure.

$\ddagger$ Values are means and standard deviations.

$\S$ Average of two values of fasting plasma glucose measured 1 week to 1 month apart. Due to missing values, sample size for baseline examination was 1218 and 775 for follow-up examination.

|| Due to missing values sample size for analysis with glycated haemoglobin as outcome was 775 .

Table 3. Partial Pearson correlations $(r)$ of participants in the Insulin Resistance and Atherosclerosis Study at baseline $(n$ 1255) and follow-up (n 813) examinations $\dagger$

\begin{tabular}{|c|c|c|c|c|}
\hline & Average fasting glucose $\ddagger$ & 2-h postprandial glucose & 2-h postprandial glucose (adjusted§) & Glycated haemoglobin\| \\
\hline \multicolumn{5}{|c|}{ Glycaemic index } \\
\hline Baseline & -0.04 & -0.04 & -0.03 & NA \\
\hline Follow-up & -0.004 & -0.01 & -0.02 & 0.01 \\
\hline \multicolumn{5}{|c|}{ Glycaemic load } \\
\hline Baseline & -0.04 & -0.01 & 0.04 & NA \\
\hline Follow-up & -0.03 & -0.02 & 0.005 & 0.01 \\
\hline \multicolumn{5}{|c|}{ Dietary carbohydrate } \\
\hline Baseline & -0.02 & 0.01 & 0.05 & NA \\
\hline Follow-up & -0.03 & -0.02 & 0.01 & -0.004 \\
\hline \multicolumn{5}{|l|}{ Dietary fibre } \\
\hline Baseline & -0.03 & -0.01 & 0.04 & NA \\
\hline Follow-up & $-0.07^{\star}$ & -0.02 & 0.03 & $-0.08^{*}$ \\
\hline \multicolumn{5}{|c|}{ Dietary starch } \\
\hline Baseline & $-0.08^{*}$ & -0.04 & 0.02 & NA \\
\hline Follow-up & -0.06 & 0.03 & $0.08^{*}$ & -0.03 \\
\hline \multicolumn{5}{|c|}{ Dietary fructose } \\
\hline Baseline & 0.03 & 0.04 & 0.04 & NA \\
\hline Follow-up & 0.03 & -0.05 & $-0.07^{\star}$ & 0.04 \\
\hline \multicolumn{5}{|c|}{ Dietary glucose } \\
\hline Baseline & 0.04 & $0.06^{*}$ & 0.05 & NA \\
\hline Follow-up & 0.04 & -0.05 & $-0.10^{*}$ & 0.05 \\
\hline
\end{tabular}

NA, not applicable.

${ }^{*} P<0.05$.

† Values adjusted for total energy intake.

$\ddagger$ Average of two values of fasting plasma glucose measured 1 week to 1 month apart. Due to missing values, sample size for baseline examination was 1218 and 775 for follow-up examination.

§ Additionally adjusted for fasting plasma glucose to indicate $2 \mathrm{~h}$ incremental glucose as outcome.

|| Due to missing values, sample size for analysis with glycated haemoglobin as outcome was 775 . 
Table 4. Generalized linear regression models with measures of glycaemia as outcome variables for participants in the Insulin Resistance Atherosclerosis Study at the baseline $(n 1255)$ and follow-up ( $n$ 813) examinations

\begin{tabular}{|c|c|c|c|c|c|c|c|c|}
\hline \multirow[b]{2}{*}{ Exposure } & \multicolumn{2}{|c|}{ Model 1† } & \multicolumn{2}{|c|}{ Model $2 \ddagger$} & \multicolumn{2}{|c|}{ Model 3§ } & \multicolumn{2}{|c|}{ Model 4\| } \\
\hline & $\beta \emptyset$ & SE & $\beta$ & SE & $\beta$ & SE & $\beta$ & SE \\
\hline \multicolumn{9}{|c|}{ Average fasting glucose $(\mathrm{mg} / \mathrm{dl})^{\star \star}$} \\
\hline \multicolumn{9}{|c|}{ Glycaemic index } \\
\hline Baseline & 0.11 & 0.84 & 0.03 & 0.89 & -0.22 & 0.84 & & \\
\hline Follow-up & 0.05 & 0.69 & -0.21 & 0.74 & -0.27 & 0.74 & & \\
\hline \multicolumn{9}{|c|}{ Glycaemic load } \\
\hline Baseline & $-4 \cdot 04^{*}$ & $2 \cdot 42$ & $-4 \cdot 04^{\star}$ & 2.42 & $-3 \cdot 23$ & $2 \cdot 42$ & & \\
\hline Follow-up & $-3 \cdot 24$ & $1 \cdot 62$ & -2.42 & 1.62 & -1.62 & 1.62 & & \\
\hline \multicolumn{9}{|c|}{ Dietary carbohydrate } \\
\hline Baseline & $-4 \cdot 02^{*}$ & 2.01 & -4.02 & 2.01 & $-2 \cdot 01$ & 2.01 & & \\
\hline Follow-up & $-3 \cdot 08^{*}$ & 2.06 & -2.06 & 2.06 & -1.03 & 2.06 & & \\
\hline \multicolumn{9}{|l|}{ Dietary fibre } \\
\hline Baseline & -1.91 & $1 \cdot 11$ & $-1 \cdot 35$ & $1 \cdot 11$ & -0.80 & $1 \cdot 11$ & & \\
\hline Follow-up & $-2.63^{*}$ & 0.99 & $-2 \cdot 05^{\star}$ & 1.07 & -1.72 & 0.99 & & \\
\hline \multicolumn{9}{|c|}{ Dietary starch } \\
\hline Baseline & -2.08 & 1.56 & -2.08 & 1.56 & -2.60 & 1.56 & & \\
\hline Follow-up & -2.61 & 1.04 & -2.08 & 1.56 & -1.56 & 1.04 & & \\
\hline \multicolumn{9}{|c|}{ Dietary fructose } \\
\hline Baseline & -0.53 & 0.88 & -0.71 & 0.88 & -0.53 & 0.88 & & \\
\hline Follow-up & -0.08 & 0.83 & 0.17 & 0.83 & 0.5 & 0.83 & & \\
\hline \multicolumn{9}{|c|}{ Dietary glucose } \\
\hline Baseline & -0.33 & 0.82 & -0.49 & 0.82 & -0.33 & 0.82 & & \\
\hline Follow-up & 0.15 & 0.77 & 0.46 & 0.77 & 0.61 & 0.77 & & \\
\hline \multicolumn{9}{|c|}{$2 \mathrm{~h}$ postprandial glucose (mg/dl) } \\
\hline \multicolumn{9}{|c|}{ Glycaemic index } \\
\hline Baseline & 1.73 & 2.01 & $2 \cdot 24$ & $2 \cdot 12$ & 1.57 & $2 \cdot 01$ & 1.29 & 1.34 \\
\hline Follow-up & $1 \cdot 80$ & 2.07 & $2 \cdot 28$ & $2 \cdot 18$ & 1.91 & $2 \cdot 12$ & 2.07 & 1.59 \\
\hline Glycaemic lo & & & & & & & & \\
\hline Baseline & -4.04 & 4.85 & -4.04 & $4 \cdot 85$ & -0.81 & 4.84 & $5 \cdot 66$ & $3 \cdot 23$ \\
\hline Follow-up & $-3 \cdot 24$ & 4.86 & -2.43 & $4 \cdot 86$ & 0.40 & $4 \cdot 86$ & 4.05 & 4.05 \\
\hline Dietary carbc & & & & & & & & \\
\hline Baseline & -5.03 & 5.03 & -6.03 & 5.03 & -2.01 & 5.03 & 4.02 & 3.02 \\
\hline Follow-up & $-6 \cdot 17$ & $5 \cdot 14$ & $-5 \cdot 14$ & $5 \cdot 14$ & -3.08 & $5 \cdot 14$ & $1 \cdot 28$ & $4 \cdot 11$ \\
\hline Dietary fibre & & & & & & & & \\
\hline Baseline & -5.65 & $2 \cdot 71$ & $-4 \cdot 14$ & $2 \cdot 79$ & -2.79 & 2.63 & -0.40 & 1.75 \\
\hline Follow-up & -4.84 & 2.96 & $-3 \cdot 78$ & 3.04 & $-2 \cdot 71$ & 2.96 & 0.99 & $2 \cdot 22$ \\
\hline Dietary starc & & & & & & & & \\
\hline Baseline & -1.56 & 3.64 & -1.04 & 3.64 & -1.56 & 3.64 & 1.56 & 2.08 \\
\hline Follow-up & 4.69 & 3.65 & 3.65 & $4 \cdot 17$ & 4.69 & 3.65 & $7 \cdot 30^{*}$ & $3 \cdot 13$ \\
\hline Dietary fructc & & & & & & & & \\
\hline Baseline & 0.53 & $2 \cdot 12$ & -0.09 & $2 \cdot 12$ & 0.53 & $2 \cdot 12$ & 1.59 & 1.42 \\
\hline Follow-up & $-4 \cdot 17$ & $2 \cdot 33$ & -4.00 & $2 \cdot 33$ & -3.33 & $2 \cdot 33$ & $-3.83^{\star}$ & 1.67 \\
\hline Dietary gluco & & & & & & & & \\
\hline Baseline & $1 \cdot 80$ & $2 \cdot 13$ & $1 \cdot 31$ & $2 \cdot 13$ & 1.64 & $2 \cdot 13$ & 2.29 & 1.31 \\
\hline Follow-up & $-4 \cdot 76^{\star}$ & $2 \cdot 3$ & -4.61 & $2 \cdot 3$ & -3.99 & $2 \cdot 3$ & $-4 \cdot 76^{\star}$ & 1.69 \\
\hline Glycated haem & \%)†† & & & & & & & \\
\hline Glycaemic in & & & & & & & & \\
\hline $\begin{array}{l}\text { Follow-up } \\
\text { Glycaemic lo }\end{array}$ & -0.01 & 0.03 & -0.002 & 0.03 & -0.003 & 0.03 & 0.01 & 0.02 \\
\hline Follow-up & -0.03 & 0.08 & -0.02 & 0.08 & 0.01 & 0.08 & 0.05 & 0.08 \\
\hline Dietary carbo & & & & & & & & \\
\hline Follow-up & -0.04 & 0.10 & -0.03 & 0.10 & -0.01 & 0.10 & 0.03 & 0.05 \\
\hline Dietary fibre & & & & & & & & \\
\hline Follow-up & $-0.08^{\star}$ & 0.04 & $-0.08^{\star}$ & 0.04 & -0.07 & 0.04 & -0.02 & 0.02 \\
\hline Dietary starc & & & & & & & & \\
\hline Follow-up & -0.05 & 0.05 & -0.05 & 0.05 & -0.05 & 0.05 & -0.01 & 0.05 \\
\hline Dietary fructc & & & & & & & & \\
\hline Follow-up & 0.01 & 0.03 & 0.02 & 0.03 & 0.02 & 0.03 & 0.01 & 0.02 \\
\hline Dietary gluco & & & & & & & & \\
\hline Follow-up & 0.02 & 0.03 & 0.02 & 0.03 & 0.03 & 0.03 & 0.01 & 0.02 \\
\hline
\end{tabular}

${ }^{*} P<0.05$.

† Adjusted for total energy intake, age, sex, ethnicity and clinic.

$\ddagger$ Adjusted for total energy intake, age, sex, ethnicity, clinic, alcohol intake, smoking status and activity.

$\S$ Adjusted for total energy intake, age, sex, ethnicity, clinic, alcohol intake, smoking status, activity and BMl.

$\|$ Adjusted for total energy intake, age, sex, ethnicity, clinic, alcohol intake, smoking status, activity, BMl, and fasting glucose or average fasting glucose if outcome is glycated haemoglobin, indicating $2 \mathrm{~h}$ incremental glucose as outcome.

I $\beta$ is change per 1 SD of exposure.

${ }^{* \star}$ Average of two values of fasting plasma glucose measured 1 week to 1 month apart. Due to missing values, sample size for baseline examination was 1218 and 775 for follow-up examination.

t†Due to missing values sample size for analysis with glycated haemoglobin as outcome was 775 . 
glucose tended to be associated at baseline $(0.05 \leq P<0 \cdot 10)$ but was inversely associated at follow-up $(P<0 \cdot 05)$. Also at followup, dietary starch was positively, and fructose was inversely, associated with $2 \mathrm{~h}$ incremental glucose (both $P<0 \cdot 05$ ). For the outcome A1c, only dietary fibre was statistically significantly, inversely, associated $(P<0.05)$ after adjustment for demographic variables and lifestyle factors (Table 4 , models 1 and 2). This association did not remain statistically significant after BMI was included in the model $(0.05 \leq P<0 \cdot 10$, Table 4 , model 4). Finally, models were adjusted for average fasting glucose, thus modelling exposures in relation to chronic glycaemia due primarily to dietary influences rather than to overnight endogenous glucose production; none of the dietary variables was significantly associated with A1c (Table 4, model 4). Further adjustment of final models (models 3 and 4) for dietary fat and protein (either separately or together) to account for possible confounding due to fat and protein in mixed diets did not materially alter the findings (data not shown).

Analyses were repeated after exclusion of individuals with previously undiagnosed diabetes. Results were essentially unchanged for GI and GL. Among the other dietary variables, dietary fibre was significantly, inversely associated with average fasting glucose (at follow-up examination), $2 \mathrm{~h}$ postprandial glucose (at both baseline and follow-up examinations), $2 \mathrm{~h}$ incremental glucose (at follow-up examination) and A1c (all $P<0.05)$. At the baseline examination dietary fibre was inversely associated with average fasting glucose and $2 \mathrm{~h}$ incremental glucose but did not reach significance $(0 \cdot 05 \leq P<0 \cdot 1)$. Dietary starch was significantly, positively associated with $2 \mathrm{~h}$ postprandial glucose at follow-up examination $(P<0 \cdot 05)$.

Analyses were repeated using linear mixed models to incorporate observations from both examinations for associations of GI and GL with measures of glycaemia (other than A1c); no statistically significant associations were detected (data not shown). From stratified analyses, neither African American participants nor Hispanic participants differed from non-Hispanic white participants in terms of associations between GI or GL and A1c. And, in formal tests of interaction terms for the outcome variable A1c, again, no differences were detected across race/ethnic groups. Primarily because we observed no statistically significant associations between GI from the assessment of usual dietary intake over the prior year and current measures of glycaemia, we did not pursue further analyses of associations between GI and measures of glycaemia at follow-up, 5 years hence.

\section{Discussion}

The major finding of these analyses was that no statistically significant association was observed for GI in relation to fasting glucose, postprandial glucose, incremental postprandial glucose or A1c; either at baseline or at the follow-up examination. Furthermore, GI was not associated with A1c after adjustment for fasting glucose. For GL, an inverse association was observed relative to fasting glucose, although statistical significance was not attained consistently between examinations (Table 4, models 1,2 and 3). GL tended to be associated with $2 \mathrm{~h}$ incremental glucose $(0.05 \leq P<0 \cdot 10$; Table 4, model 4) at baseline but not at the follow-up examination. Findings for GL were very closely mirrored by findings for total carbohydrate, which may be accounted for by the high correlation between GL and total carbohydrate, after adjustment for total energy intake ( $r 0.89$ and 0.92 at baseline and follow-up, respectively). We did observe several statistically significant associations of other carbohydrate-related variables in relation to measures of glycaemia, particularly for dietary fibre; however, these tended to be small in magnitude and inconsistent between the baseline and follow-up examinations.

The underlying rationale for our expectation that GI of the usual diet would be directly associated with A1c arises from the underlying rationale for the use of acarbose to prevent or treat type 2 diabetes. Acarbose, an $\alpha$-glucosidase inhibitor, inhibits amylase and sucrase enzyme activity and thus slows carbohydrate digestion. In a dose-dependent fashion, acarbose decreases postprandial glucose and insulin excursions, and via reduced hyperinsulinaemia, triacylglycerol uptake into adipose tissue and hepatic lipogenesis is reduced (Bischoff, 1995). In human subjects with diabetes, acarbose has been shown to reduce A1c levels substantially (approximately 0.7 to $>1 \%$ ) and in a dose-dependent manner (Coniff et al. 1995). This general pathway is essentially that which has been proposed as the principle underlying GI, namely that slowed digestion and absorption of carbohydrate from food, accomplished via choice of low-GI foods, will similarly yield long-term metabolic benefits initiated by blunted glucose excursions following consumption of carbohydratecontaining foods (Wolever, 1990).

Studies of GI of test meals have yielded results that would support the utility of this index to capture the multitude of factors that contribute to digestion and absorption rates of carbohydrate-containing foods, and predict glucose response to foods (Wolever et al. 1991). Recently, the 'physiological validity' of the concept of GL of breakfast meals, in which variation in GL in the test meals was associated with plasma glucose measured $2 \mathrm{~h}$ after ingestion of the test meals (Brand-Miller et al. 2003), was demonstrated.

Whether the results of these short-term studies can be translated to use of GI or GL in the study of long-term health effects of a usual diet is uncertain. Findings of the present report would suggest that the simple incorporation of GI or GL into an assessment of usual dietary intake from a validated food frequency instrument may, in fact, have little utility relative to the original concept of GI. And, GL appears to provide little information beyond total carbohydrate content of the diet. This is not to say that GI, as currently quantified, does not provide some indication of diet quality. Schulz et al. (2004) have recently described many strong correlations of GI and GL with a variety of nutrients and foods, also from the IRAS FFQ. GI and GL of usual diet was associated with total energy intake ( $r 0.25$ and 0.91 , respectively, $P<0.001)$. After adjustment for total energy intake, GI was inversely associated with fructose, glucose, sucrose and fibre; positively associated with starch; inversely associated with protein; and positively associated with dietary fat (total and major fat subtypes) (all $P<0 \cdot 001$ ). White bread, beer, meats and fries/ fried potatoes were positively associated with GI, and intake of fruits and low-fat milk was inversely associated with GI (all $P<0.001$ ); as would be expected.

Previous studies suggest that usual dietary intake may be related to A1c. In a study of nearly 10000 individuals, those who took sugar with tea or coffee, those who used solid fat for cooking and those who drank whole milk (rather than 
reduced-fat milk) had higher A1c than those without these dietary habits; however, A1c did not vary with intake of dried beans and peas, fruits, vegetables and salads, cakes or sweets (Gulliford \& Ukoumunne, 2001). GI from $3 \mathrm{~d}$ food diaries was associated positively with $\mathrm{A} 1 \mathrm{c}$ in a large cohort of individuals with type 1 diabetes (Buyken et al. 2001); however, use of insulin in this population makes this finding difficult to interpret relative to the aim of the present study.

There are several limitations of the present work that may have contributed to the lack of a statistically significant association of GI or other carbohydrate-related exposure variables with A1c in the present study (other than the significant association observed for fibre intake except in the final model). First, the removal of more metabolically vulnerable individuals in the follow-up examination of those individuals who either failed to return for that examination, and of those who advanced (especially from impaired glucose tolerance) to type 2 diabetes by the time of the follow-up examination may have unduly restricted the range of $\mathrm{A} 1 \mathrm{c}$ required to detect the effect of interest. Second, although the IRAS FFQ has been validated in a subsample of the IRAS cohort (Mayer-Davis et al. 1999b), this FFQ was not constructed originally for the purpose of capturing GI, and GI values are not available in the nutrient database used in the validation study (Nutrition Coordinating Center, 1990). Thus the validity of the GI values generated by the IRAS FFQ is unknown, and this information has not been published from other similar FFQ to which GI has been added. Nonetheless, the mean GI in IRAS was essentially identical to GI values from the NHANES study (Ford \& Liu, 2001), and the IRAS FFQ has yielded statistically significant associations between various nutrients and food groups, and health outcomes (Mayer-Davis et al. 1997,1999a; Bell et al. 2000; Liese et al. 2003, 2004). A third potential limitation was sample size, given that some previously published positive results for an association of GI with diabetes incidence were conducted in study samples far larger than that available from IRAS (Salmeron et al. 1997a,b). Given the sample size of 775 for A1c at the second examination and the standard deviations of A1c and GI in the present dataset, a correlation coefficient of 0.013 could be detected with $80 \%$ power; thus, the study had adequate statistical power to reasonably expect to detect a statistically significant association between GI and A1c, if one truly existed. For other measures of glycaemia evaluated, data were re-analysed to maximize precision in estimates of association by use of data from both examinations in a linear mixed model. Still no statistically significant associations were observed between GI and the outcomes. Finally, there may have been insufficient exposure to low GI diets in the IRAS population to detect a true association between GI and measures of glycaemia.

Despite these limitations, we suggest that the lack of association reported herein between dietary GI and any measure of glycaemia, together with the inconsistencies in the published literature for the effect of dietary GI on diabetes incidence, may be due primarily to inadequate utility of the GI to capture the true metabolic impact of foods consumed as part of a usual diet. Similarly, inconsistencies in the present report regarding associations of carbohydrate-related nutrient variables with measures of glycaemia may reflect inadequate capture by these specific nutrient variables of the overall impact of carbohydrate-containing foods on chronic glycaemia. As summarized by Gannon \& Nuttall (1987), it remains true today that considerable methodological work will be needed to generate a dietary measure or set of measures that capture the known nutrient and non-nutrient determinants of carbohydrate digestion and absorption rates, and their metabolic effects. Such measures should incorporate quantifiable characteristics of food so that the underlying effects of foods can be better understood. For example, the eventual (post-absorption) glucose content of the food, the ratio of amylopectin: amylase of starch-containing foods, and cooking times, or other food processing that substantially affects the digestibility of starch may need to be incorporated into this measure. It has been suggested that the glucose excursions should be quantified based on glucose excursion through the time required for glucose to return to the basal state, rather than on a more limited or predetermined time period (Gannon \& Nuttall, 1987; Nuttall \& Gannon, 1999). In addition, the amount of time required for glucose (and perhaps insulin) (Gannon \& Nuttall, 1987; Nuttall \& Gannon, 1991, 1999; Holt et al. 1997) to return to basal levels following ingestion of a food alone or in a mixed meal, as well as time-related patterns of sequential meals and snacks, may be important to quantify. In summary, we suggest that the concept of GI has allowed development of important hypotheses regarding the impact of food intake on glucose excursion and associated metabolic parameters. However, considerable methodological work is now required to further our understanding of the specific contributors of usual intake of carbohydrate-containing foods on these metabolic parameters, and on relevant disease states.

\section{Acknowledgements}

The present study was supported by a Clinical Research Grant from the American Diabetes Association to Dr Angela Liese, and by NIH/NHLBI grants UO1 HL/17 887, UO1 HL/17 889, UO1 HL/17 890, UO1 HL/17 892, UO1 HL/17902 and DK29867. Each author contributed substantively to the work including critical review of the manuscript. Dr Mayer-Davis conceived the basic premise and analytic approach for the paper, directed the implementation of the FFQ in IRAS and led the writing of the paper. Dr Dhawan conducted the statistical analyses and contributed substantially to the writing; Dr Liese directed the application of GI and GL to the IRAS FFQ; Dr Teff provided substantial advice on underlying physiology; Ms Schulz conducted technical work to apply GI and GL to the IRAS FFQ. None of the authors had any personal interest in any company or organization sponsoring the research.

\section{References}

Bell RA, Mayer-Davis EJ, Martin MA, D’Agostino RB Jr \& Haffner SM (2000) Associations between alcohol consumption and insulin sensitivity and cardiovascular disease risk factors: the Insulin Resistance and Atherosclerosis Study. Diabetes Care 23, $1630-1636$.

Bischoff H (1995) The mechanism of alpha-glycosidase inhibition in the management of diabetes. Clin Invest Med 18, 303-311.

Block G, Hartman AM, Dresser CM, Carroll MD, Gannon J \& Gardner L (1986) A data-based approach to diet questionnaire design and testing. Am J Epidemiol 124, 453-469.

Brand-Miller JC, Thomas M, Swan V, Ahmad ZI, Petocz P \& Colagiuri S (2003) Physiological validation of the concept of glycemic load in lean young adults. $J$ Nutr 133, 2728-2732. 
Buyken AE, Toeller M, Heitkamp G, Karamanos B, Rottiers R, Muggeo M \& Fuller JH (2001) Glycemic index in the diet of European outpatients with type 1 diabetes: relations to glycated hemoglobin and serum lipids. Am J Clin Nutr 73, 574-581.

Coniff RF, Shapiro JA, Robbins D, Kleinfield R, Seaton TB, Beisswenger P \& McGill JB (1995) Reduction of glycosylated hemoglobin and postprandial hyperglycemia by acarbose in patients with NIDDM. A placebo-controlled dose-comparison study. Diabetes Care 18, 817-824.

Coniff RF, Shapiro JA \& Seaton TB (1994) Long-term efficacy and safety of acarbose in the treatment of obese subjects with non-insulin-dependent diabetes mellitus. Arch Intern Med 154, $2442-2448$.

Eckel RH (2003) A new look at dietary protein in diabetes. Am J Clin Nutr 78, 671-672.

Ercan N, Gannon MC \& Nuttall FQ (1994) Effect of added fat on the plasma glucose and insulin response to ingested potato given in various combinations as two meals in normal individuals. Diabetes Care 17, 1453-1459.

Ford ES \& Liu S (2001) Glycemic index and serum high-density lipoprotein cholesterol concentration among US adults. Arch Intern Med 161, 572-576.

Foster-Powell K, Holt SH \& Brand-Miller JC (2002) International table of glycemic index and glycemic load values: 2002. Am J Clin Nutr 76, 5-56.

Gannon MC \& Nuttall FQ (1987) Factors affecting interpretation of postprandial glucose and insulin areas. Diabetes Care 10, $759-763$.

Gannon MC \& Nuttall FQ (2004) Effect of a high-protein, low-carbohydrate diet on blood glucose control in people with type 2 diabetes. Diabetes 53, 2375-2382.

Gannon MC, Nuttall FQ, Saeed A, Jordan K \& Hoover H (2003) An increase in dietary protein improves the blood glucose response in persons with type 2 diabetes. Am J Clin Nutr 78, 734-741.

Gulliford MC \& Ukoumunne OC (2001) Determinants of glycated haemoglobin in the general population: associations with diet, alcohol and cigarette smoking. Eur J Clin Nutr 55, 615-623.

Hanley AJ, D'Agostino R Jr, Wagenknecht LE, Saad MF, Savage PJ, Bergman R \& Haffner SM (2002) Increased proinsulin levels and decreased acute insulin response independently predict the incidence of type 2 diabetes in the Insulin Resistance Atherosclerosis Study. Diabetes 51, 1263-1270.

Hodge AM, English DR, O'Dea K \& Giles GG (2004) Glycemic index and dietary fiber and the risk of type 2 diabetes. Diabetes Care 27, 2701-2706.

Holt SH, Miller JC \& Petocz P (1997) An insulin index of foods: the insulin demand generated by $1000-\mathrm{kJ}$ portions of common foods. Am J Clin Nutr 66, 1264-1276.

Jenkins DJ, Jenkins AL, Wolever TM, Vuksan V, Rao AV, Thompson LU \& Josse RG (1994) Low glycemic index: lente carbohydrates and physiological effects of altered food frequency. Am J Clin Nutr 59, 706S-709S.

Jenkins DJ, Wolever TM, Taylor RH, Barker H, Fielden H, Baldwin JM, Bowling AC, Newman HC, Jenkins AL \& Goff DV (1981) Glycemic index of foods: a physiological basis for carbohydrate exchange. Am J Clin Nutr 34, 362-366.

Liese AD, Roach AK, Sparks KC, Marquart L, D'Agostino RB Jr \& Mayer-Davis EJ (2003) Whole-grain intake and insulin sensitivity: the Insulin Resistance Atherosclerosis Study. Am J Clin Nutr 78, 965-971.

Liese AD, Schulz M, Moore CG \& Mayer-Davis EJ (2004) Dietary patterns, insulin sensitivity and adiposity in the multi-ethnic Insulin Resistance Atherosclerosis Study population. Br J Nutr 92, 973-984.

Littell RC, Pendergast J \& Natarajan R (2000) Modeling covariance structure in the analysis of repeated measures data. Stat Med 19, $1793-1819$.
Mayer-Davis EJ, D’Agostino R Jr, Karter A, Haffner S, Rewers M, Saad MF \& Bergman RN for the IRAS investigators (1998) Intensity and amount of physical activity in relation to insulin sensitivity. The Insulin Resistance Atherosclerosis Study (IRAS). JAMA 279, 669-674.

Mayer-Davis EJ, Levin S \& Marshall JA (1999a) Heterogeneity in associations between macronutrient intake and lipoprotein profile in individuals with type 2 diabetes. Diabetes Care 22, 1632-1639.

Mayer-Davis EJ, Monaco JH, Hoen HM, et al. (1997) Dietary fat and insulin sensitivity in a triethnic population: the role of obesity. The Insulin Resistance Atherosclerosis Study (IRAS). Am J Clin Nutr 65, 79-87.

Mayer-Davis EJ, Vitolins MZ, Carmichael SL, Hemphill S, Tsaroucha G, Rushing J \& Levin S (1999b) Validity and reproducibility of a food frequency interview in a Multi-Cultural Epidemiology Study. Ann Epidemiol 9, 314-324.

Meyer KA, Kushi LH, Jacobs DR Jr, Slavin J, Sellers TA \& Folsom AR (2000) Carbohydrates, dietary fiber, and incident type 2 diabetes in older women. Am J Clin Nutr 71, 921-930.

Nutrition Coordinating Center (1990) Minnesota Nutrition Data System. Program 2.3, Food Database Version 2.2, Nutrient Database Version 19. Minneapolis: University of Minnesota.

Nuttall FQ \& Gannon MC (1991) Plasma glucose and insulin response to macronutrients in nondiabetic and NIDDM subjects. Diabetes Care 14, 824-838.

Nuttall FQ \& Gannon MC (1999) Carbohydrates and diabetes. In American Diabetes Association Guide to Medical Nutrition Therapy for Diabetes, pp. 85-106 [MJ Franz and JP Bantle, editors]. Alexandria, VA: American Diabetes Association.

Nuttall FQ, Gannon MC, Wald JL \& Ahmed M (1985) Plasma glucose and insulin profiles in normal subjects ingesting diets of varying carbohydrate, fat, and protein content. J Am Coll Nutr 4, 437-450.

Perneger TV (1998) What's wrong with Bonferroni adjustments 1. Br Med J 316, 1236-1238.

Salmeron J, Ascherio A, Rimm EB, Colditz GA, Spiegelman D, Jenkins DJ, Stampfer MJ, Wing AL \& Willett WC (1997b) Dietary fiber, glycemic load, and risk of NIDDM in men. Diabetes Care 20, $545-550$.

Salmeron J, Manson JE, Stampfer MJ, Colditz GA, Wing AL \& Willett WC (1997a) Dietary fiber, glycemic load, and risk of non-insulin dependent diabetes in women. JAMA 277, 472-477.

Schulz M, Liese AD, Fang F, Mayer-Davis EJ, Sparks KC \& Wolever TMS (2004) Nutritional correlates of glycemic index: American Diabetes Association 64th Scientific Sessions. Diabetes 53, Suppl. 2, A91.

Schulze MB, Liu S, Rimm EB, Manson JE, Willett WC \& Hu FB (2004) Glycemic index, glycemic load, and dietary fiber intake and incidence of type 2 diabetes in younger and middle-aged women. Am J Clin Nutr 80, 348-356.

Stevens J, Ahn K, Juhaeri, et al. (2002) Dietary fiber intake and glycemic index and incidence of diabetes in African-American and white adults: the ARIC study. Diabetes Care 25, 1715-1721.

Tahara Y \& Shima K (1995) Kinetics of HbA1c, glycated albumin, and fructosamine and analysis of their weight functions against preceding plasma glucose level 1. Diabetes Care 18, 440-447.

Wagenknecht LE, Mayer EJ, Rewers M, et al. (1995) The Insulin Resistance Atherosclerosis Study (IRAS): objectives, design, and recruitment results. Ann Epidemiol 5, 464-472.

Wolever TM (1990) The glycemic index. World Rev Nutr Diet 62 , $120-185$.

Wolever TM, Jenkins DJ, Jenkins AL \& Josse RG (1991) The glycemic index: methodology and clinical implications. Am J Clin Nutr 54, 846-854.

World Health Organization (1985) Diabetes Mellitus: Report of a WHO Study Group. Technical Report Series no. 727. Geneva: WHO. 